
\title{
25 Research Soure \\ Effect of MYH10 Variant on Acute Leukemia with Low Platelet Count Prognosis
}

\section{Qirong Xiao}

Fujian Medical University

\section{Bicun Lin}

Fujian Medical University

\section{Kaiting Lin}

Fujian Medical University

\section{Xiaobin Lin}

Fujian Medical University

ping chen ( $\sim$ pingchen0428@163.com )

Fujian Medical University Union Hospital https://orcid.org/0000-0002-0737-4991

\section{Research}

Keywords: acute myeloid leukemia, platelets, prognosis, MYH10, bioinformatics

Posted Date: September 21st, 2021

DOI: https://doi.org/10.21203/rs.3.rs-895701/v1

License: (c) (1) This work is licensed under a Creative Commons Attribution 4.0 International License. Read Full License 


\section{Abstract}

Background: Acute myeloid leukemia (AML) is a blood disorder characterized by abnormal white blood cell count, anemia, or abnormal platelet count. It is associated with molecular genetic changes. While platelet counts vary at first diagnosis platelets recover after chemotherapy.

Objectives:This study aimed to; (1) Investigate whether the platelet count and genotype at first diagnosis are related to chemotherapy and their significance on chemotherapy prognosis; (2) Determine whether newly diagnosed patients with low platelet count have a poor prognosis and if it can be used as a separate prognostic predictor; (3) Determine whether the mutation genotype affects platelet count and its prognosis at first diagnosis.

Methods: A retrospective chart review of $301 \mathrm{AML}$ patients was conducted. Univariate, multivariate unconditional Logistic regression and Cox regression analyses were also conducted. Bioinformatics technology was used to extract the GSE12662 data set from the GEO database to analyze differentially expressed genes in AML patients. Besides, biological functions, pathways, proteins, and prognosis were assessed.

Conclusion: Increased platelet count in AML patients after chemotherapy was an independent risk factor affecting complete remission. The platelet count also had some guiding significance for evaluating the sensitivity of patients to chemotherapy. MYH10 causes thrombocytopenia in acute myeloid leukemia via RUNX1 gene alteration and influence of prognostic factors. MYH10 variant detection improves the identification of $A M L$ molecular characteristics and its prognostic impact on AML, helping in the response analysis to chemotherapeutic agents and further treatment decisions.

\section{Introduction}

Leukemia is a hematopoietic malignancy with a poor prognosis and high mortality ${ }^{1}$. Blood system disorders, such as abnormal white blood cell counts, anemia, or abnormal platelet count, are the most common acute leukemia characteristics. Studies have shown that chemotherapy is associated with longterm prognosis in acute myeloid leukemia (AML) patients. Patients with early complete remission (CR) have a longer survival period ${ }^{2-5}$. The molecular genetic characteristics of AML patients at the onset vary with age ${ }^{6-9}$, and the molecular genetics of good prognosis gradually decline with AML onset. However, the proportion of gene or chromosomal mutations that mark poor prognosis gradually increases. Several studies have shown that the degree of blood cell recovery in CR patients (morphological leukemia-free state (MLFS) with the recovery of absolute neutrophil count (ANC) and platelets (PLT)) is associated with prognosis.

Moreover, the treatment results are significantly better than those of low blood cells ${ }^{10-14}$. However, the effect of platelet count on AML prognosis at the initial diagnosis and after chemotherapy is unknown. Therefore, in this study, 301 newly-treated AML cases (except M3) were retrospectively analyzed in the 
institute to explore the influence of platelet count and related factors on prognosis at the initial diagnosis and after chemotherapy. Biological information was also used to examine the impact of molecular genetic changes on the newly diagnosed platelet count and its prognosis.

\section{Results}

1.1 Impact of platelet count during initial treatment

The relevant information and clinical indicators of the 301 newly-treated AML patients were collected (Table 1). 
Table 1

Basic characteristics of primary AML patients $(\mathrm{N}=301)$

\begin{tabular}{|c|c|c|}
\hline \multicolumn{2}{|l|}{ Characteristics } & \multirow{2}{*}{$\begin{array}{l}(\%) \\
238(79.1)\end{array}$} \\
\hline Age(year) & $\leqq 55$ & \\
\hline & $56-74$ & $58(19.2)$ \\
\hline & $\geq 75$ & $5(1.7)$ \\
\hline \multirow[t]{2}{*}{ Sex } & Male & $167(55.5)$ \\
\hline & Female & $134(44.5)$ \\
\hline \multirow[t]{4}{*}{ Risk } & Low-risk & 112(37.2) \\
\hline & Medium-risk & $54(17.9)$ \\
\hline & High-risk & $128(42.5)$ \\
\hline & Unknown & $7(2.4)$ \\
\hline \multirow[t]{2}{*}{ Hemorrhage } & Yes & $124(41.2)$ \\
\hline & No & 117(38.8) \\
\hline \multirow[t]{4}{*}{ Infected } & One month before chemotherapy & $122(40.5)$ \\
\hline & During Chemotherapy & $158(42.5)$ \\
\hline & Six month after chemotherapy & 48(15.9) \\
\hline & No & $3(1.1)$ \\
\hline \multirow[t]{3}{*}{ Chemotherap } & High dose cytarabine & $4(1.3)$ \\
\hline & Low dose cytarabine & $14(4.7)$ \\
\hline & $3+7$ regimen & $238(94.0)$ \\
\hline \multirow[t]{3}{*}{ Platelet reco } & Yes & $129(42.9)$ \\
\hline & No & $169(56.1)$ \\
\hline & Unchanged & $3(1.0)$ \\
\hline \multirow[t]{2}{*}{ Platelet trans } & Yes & 282(93.7) \\
\hline & No & $19(6.3)$ \\
\hline \multirow[t]{4}{*}{ Prognosis } & Complete remission & $166(55.1)$ \\
\hline & Partial remission & $39(13.0)$ \\
\hline & No remission & $79(26.3)$ \\
\hline & Death & $22(5.6)$ \\
\hline
\end{tabular}


The univariate analysis of leukemia remission and clinical indicators showed that bleeding in AML patients, platelet transfusion during chemotherapy, and platelet rise after chemotherapy are the factors that affect complete remission after chemotherapy (Table 2). 
Table 2

Correlation between $\mathrm{CR}$ and clinical characteristics in AML patients

\begin{tabular}{|c|c|c|c|c|c|c|}
\hline & Remission & & & & & \\
\hline Variables & No & $\%$ & Yes & $\%$ & $x^{2}$ & $P$ \\
\hline & $(n=135)$ & & $(n=166)$ & & & \\
\hline Sex & & & & & 1.414 & 0.234 \\
\hline Female & 55 & 40.7 & 79 & 47.6 & & \\
\hline Male & 80 & 59.3 & 87 & 52.4 & & \\
\hline Age & & & & & 4.344 & 0.114 \\
\hline$\leqq 55$ & 100 & 74.1 & 138 & 83.1 & & \\
\hline $56-74$ & 32 & 23.7 & 27 & 16.3 & & \\
\hline$\geq 75$ & 3 & 2.2 & 1 & 0.6 & & \\
\hline Risk & & & & & 3.345 & 0.341 \\
\hline Low-risk & 44 & 32.6 & 68 & 41 & & \\
\hline Medium-risk & 23 & 17 & 31 & 18.7 & & \\
\hline High-risk & 65 & 57.1 & 63 & 38 & & \\
\hline Unknown & 3 & 2.2 & 4 & 2.4 & & \\
\hline Infected & & & & & 3.468 & 0.325 \\
\hline One month before chemotherapy & 57 & 42.2 & 61 & 36.7 & & \\
\hline During chemotherapy & 38 & 28.1 & 56 & 33.7 & & \\
\hline Six month after chemotherapy & 35 & 25.9 & 47 & 28.3 & & \\
\hline No & 5 & 3.7 & 2 & 1.2 & & \\
\hline Hemorrhage & & & & & 4.115 & 0.043 \\
\hline Yes & 47 & 34.8 & 77 & 46.4 & & \\
\hline No & 88 & 65.2 & 89 & 53.6 & & \\
\hline Platelet transfusion & & & & & 4.555 & 0.033 \\
\hline Yes & 122 & 90.4 & 160 & 96.4 & & \\
\hline No & 13 & 9.6 & 6 & 3.6 & & \\
\hline Platelet recovery & & & & & 41.483 & 0.001 \\
\hline
\end{tabular}




\begin{tabular}{|c|c|c|c|c|c|c|}
\hline & \multicolumn{6}{|c|}{ Remission } \\
\hline Yes & 79 & 58.5 & 150 & 90.4 & & \\
\hline No & 56 & 41.5 & 16 & 9.6 & & \\
\hline Chemotherapy regimen & & & & & 1.082 & 0.582 \\
\hline High dose cytarabine & 2 & 1.5 & 2 & 1.2 & & \\
\hline Low dose cytarabine & 70 & 51.9 & 96 & 57.8 & & \\
\hline $3+7$ regimen & 63 & 46.7 & 68 & 41 & & \\
\hline
\end{tabular}

The multiple regression analysis results showed a relationship between complete remission and clinical indicators. A logistic multiple regression analysis was performed using the associated factors screened in the above-mentioned single factor analysis. The results indicated that complete remission in $\mathrm{AML}$ patients caused the increase in platelets after chemotherapy. Besides independent risk factors, platelet increased after chemotherapy, and the patient prognosis was better (Table 3).

Table 3

Logistic multiple regression analysis of $\mathrm{CR}$ in AML patients

\begin{tabular}{|lllll|}
\hline Variables & Bcoefficient & OR $(95 \% \mathrm{Cl})$ & $\mathrm{P}$ & \\
& Hemorrhage & & & \\
\hline No & & 1 & \\
\hline Yes & 0.442 & $1.556(0.935-2.59)$ & 0.089 \\
\hline Platelet transfusion & & & \\
\hline No & & 1 & 0.284 \\
Yes & 0.597 & $1.817(0.61-5.417)$ & \\
\hline Platelet recovery & & & \\
\hline No & & 1 & $<0.001$ \\
\hline Yes & 1.862 & $6.437(3.443-12.033)$ & $<.001$ \\
\hline Constant & -1.969 & 0.14 & \\
\hline
\end{tabular}

After a chemotherapy course, AML patients were divided into four groups based on platelet rise. Cox regression analysis was used to analyze the correlation between platelet rise and remission of AML patients after chemotherapy ( 49 days limit). (Fig. 1) The remission rate of AML patients increased with platelet rise after chemotherapy.

1.2 Cytogenetic/mutation effects at initial diagnosis 
The patients were divided into the non-mutation group, FLT3-ITD positive mutation group, and other mutation groups based on gene mutation, and into high $(>40 \times 10 / \mathrm{I})$ and low platelet groups $(\leq 40 \times 10)$ according to the platelet count /I) for analysis. The FLT3-ITD positive mutation group had a more significant effect on the chemotherapy outcome than other mutation groups $(p<0.05)$. However, the FLT3-ITD positive mutation and low platelet coexistence had the worst chemotherapy outcome $(p<0.05)$. Besides, GeneCards (https://www.genecards.org/) indicated that the FLT3-ITD gene is associated with platelets and leukemia. Therefore, the association between FLT3-ITD mutations, platelets, and the factors affecting chemotherapy outcome was investigated.

\subsection{Data quality assessment and DEG identification}

Principal component analysis (PCA) and Heat map were used for probe pretreatment in the FLT3-ITD positive group (relative to the normal group) and the FLT3-ITD negative group (relative to the normal group) from the GSE12662 (Fig. 2A, 2B)to reveal the distribution of gene expression data in each group (Fig. 2C, 2D, 2E, 2F). The GEO2R online tool was used to detect 246 and 291 differentially expressed genes from the FLT3-ITD positive group and FLT3-ITD negative group (adj.P $<0.01,|\log F C|>1$ ). The volcano map was used to visualize the differentially expressed genes in the positive and negative groups (Fig. 3A and Fig. 3B). Venn analysis is performed on the differentially expressed genes in the positive and the negative. A total of 67 common differentially expressed genes were identified (Fig. 3C). A heat map was constructed to visualize the 67 differentially expressed genes (Fig. 3D). A PPI network of 62 differentially expressed genes was constructed to show potential gene interactions via the STRING database. Three gene sets were central genes, one set included MYH10, MY05C, PLS3, PBX1, and MECOM, another set had VPREB1, RAG1, and VPREB3 and another set had LIMCH1 and TRIP6 (Fig. 4).

\subsection{GO function and KEGG pathway enrichment analysis}

GO and KEGG enrichment analyses were performed using two array data to identify the most relevant biological processes (BP), molecular functions (MF), and cellular components (CC). The representative top $100 \mathrm{GO}$ items were then extracted. The distribution of cellular components and molecular functions in the main branches of the ontology was in GO biological processes. The enrichment results showed that the 246 differentially expressed genes were positively regulated via astrocyte differentiation in BP, AT DNA in MF, actin filament bundles in CC. Furthermore, the Apelin signaling pathway in KEGG was the most enriched (Fig. 5A). The 291 differentially expressed genes were associated with the "de novo" IMP biosynthesis process in BP, the lysine $\mathrm{N}$-acetyltransferase activity in MF, acting on acetyl phosphate as a donor, and the aDNA polymerase in CC: primer complex. The metabolic enrichment of glycine, serine, and threonine in KEGG was the most abundant (Fig. 5B).

The KEGG pathways of tumors or leukemia were screened for comparative analysis. A total of $13 \mathrm{KEGG}$ pathways were identified in the two data sets, including two KEGG pathways, cancer pathways (MECOM, CDH1, IL12RB2), and transcriptional disorders in cancer (PBX1) (Fig. 5C, 5D, 5F). The GSEA method was used to reconfirm the KEGG results. Both the GSEA and the WebGestalt results showed cancer pathways (Table 4). 
Table 4

Enrichment results of KEGG pathway by GSEA

\begin{tabular}{|c|c|c|c|c|c|c|c|c|c|c|}
\hline & $\begin{array}{l}\text { GS follow } \\
\text { link to } \\
\text { MSigDB }\end{array}$ & SIZE & ES & NES & $\begin{array}{l}\text { NOM } \\
\text { p-val }\end{array}$ & $\begin{array}{l}\text { FDR } \\
\text { q-val }\end{array}$ & $\begin{array}{l}\text { FWER } \\
\text { p-val }\end{array}$ & $\begin{array}{l}\text { Rank } \\
\text { at } \\
\text { max }\end{array}$ & $\begin{array}{l}\text { Leading } \\
\text { edge }\end{array}$ & Rank \\
\hline FLT3+ & $\begin{array}{l}\text { Pathways } \\
\text { in cancer }\end{array}$ & 319 & 0.29 & 1.36 & 0 & 0.356 & 0.93 & 2336 & $\begin{array}{l}\text { tags }= \\
16 \%, \text { list } \\
=11 \%, \\
\text { signal = } \\
17 \%\end{array}$ & 29 \\
\hline FLT3- & $\begin{array}{l}\text { Pathways } \\
\text { in cancer }\end{array}$ & 319 & -0.18 & -0.89 & 0.709 & 0.762 & 1 & 4609 & $\begin{array}{l}\text { tags }= \\
27 \% \text {, list } \\
=21 \% \text {, } \\
\text { signal = } \\
34 \%\end{array}$ & 64 \\
\hline
\end{tabular}

2.3 MYH10 protein can be used as a biomarker for changes in platelet numbers in acute myeloid leukemia

Venn analysis was used to analyze the related genes in the KEGG pathway. MECOM, CDH1, and IL12RB2 were the co-genes of cancer pathways, and PBX1 a co-gene of transcription disorders in cancer. (Fig. 5D, $5 F)$. The PPI network showed that MECOM and PBX1 genes were grouped in MYH10.

Megakaryocytes (MKs) fragmentation forms platelets. The regulation of platelet production depends on the number and size of MKs. MK differentiates into polyploidy at a late stage to increase the cytoplasmic quality of each MK, resulting in huge cells ${ }^{15-17}$. MK polyploidy is characterized by cytokinesis failure caused by defects in contractile force at the cleavage groove ${ }^{18}$. Immature MKs express two types of myosin II( myosin heavy chain IIA (MYH9) and myosin heavy chain IIB (MYH10)), while mature MKs only express myosin IIA ${ }^{19}$. During MK production, myosin IIB accumulates in the cutting groove instead of myosin IIA. However, during polyploidization, the MYH10 gene is silenced, and no myosin II accumulation occurs. MK doubling and maturation occur via RUNX1-mediated non-myosin heavy chain IIB (MYH10) silencing ${ }^{19}$. MYH10 expression is silenced during the doubling and maturation process due to the negative regulation of the MYH10 gene-mediated via RUNX1. Moreover, the RUNX1 gene knockout in MKs causes thrombocytopenia ${ }^{20}$. Therefore, MYH10 and RUNX1 are involved in MK doubling and platelet production.

MYH10 and RUNX1 assessment in STRING showed that they are related to MK doubling and platelet production. The GEPIA database was also used to compare MYH10, FLT3, and RUNX1 mRNA expressions in leukemic bone marrow (LAML) and normal bone marrow. The expression levels of FLT3 and RUNX1 were higher in leukemic bone marrow than normal tissues, while MYH10 expression was lower than normal bone marrow (Fig. 6). 
In conclusion, MYH10 expression influences platelet production, indicating that MYH10 protein in platelets can be used as a marker to screen hematological malignancies related to RUNX1 gene changes, including familial platelet diseases, which tend to develop into acute myeloid leukemia (FPD / AML; OMIM 601399) ${ }^{21}$, primary or secondary AML and chronic myeloid leukemia (CML) ${ }^{22}$.

2.4 MYH10 causes thrombocytopenia and prognostic factors in acute myeloid leukemia via RUNX1 gene alteration

Bluteau ${ }^{23}$ compared the MKs between FPD/AML patients and healthy individuals and found that MYH10 mRNA was significantly increased in FPD/AML patients $(P<0.05)$. MYH10s were more in mature MKs of all FPD/AML patients compared with normal MKs. MYL9 and MYH9 expressions decreased, while MYH10 expression increased in FPD/AML MKs. However, the MYH10 protein was in the cytoplasm of mature MKs.Together with the Antony-Debre study ${ }^{24}$, RUNX1 knockdown is associated with megakaryocyte ploidy decrease and MYH10 expression increase in humans/mice. The MYH10 protein expression in the platelets of $\mathrm{CML}$ patients was examined. MYH10 protein expression was detected in the platelets of eight patients, and RUNX1 mutation in five of the eight patients. All patients with negative platelet MYH10 had negative RUNX1 mutation.

Interestingly, the platelet numbers in the eight CML patients were lower than other patients. Therefore, the GEPIA database was used to compare the effects of MYH10, RUNX1, and FLT3 genes on the OS prognosis. High FLT3 and low MYH10 expressions were associated with poor prognosis. RUNX1 did not affect the prognosis. A correction analysis between MYH10/FLT3 and MYH10/RUNX1 was also conducted. The low MYH10 expression was associated with a poor prognosis (Fig. 7).

Therefore, MYH10 causes thrombocytopenia in acute myeloid leukemia via RUNX1 gene alteration and influences prognostic factors. MYH10 variant detection improves the identification of AML molecular characteristics and its prognostic impact on AML.

\section{Discussion}

In recent years, several studies have shown a negative correlation between platelet count and OS before treatment, which can be used as an independent predictor of AML prognosis ${ }^{25-30}$. Other factors related to initial platelets, including rebound thrombocytosis, platelet production, and platelet recovery time after platelet induction chemotherapy, are independent prognostic predictors of AML or acute lymphocytic leukemia (ALL) Index ${ }^{31-33}$.

Presently, age, total WBC numbers, time to CR, cellular and molecular genetic changes are the factors affecting AML prognosis. However, the prognostic-related risk factors in this study included bleeding, platelet transfusion, and platelet count increase after chemotherapy.

In the past, age was the key factor influencing AML prognosis. Older patients had a poor prognosis due to poor physical conditions and high-risk molecular genetic phenotypes ${ }^{34}$. The average age of AML patients 
in this study was 41.2 years ( $\leq 55$ years old $(n=238) ; 56-75$ years old $(n=58)$; and $\geq 75$ years old $(n=$ $5)$ ). Age did not influence relief after the chemotherapy course, possibly because newly-treated AML patients admitted to the hospital were under 55 years, and the number of older patients was small. However, further research is needed to clarify the specific cause.

Studies have shown that infection promotes IL-6 and thrombopoietin production in the body and liver, respectively, thereby promoting platelet production ${ }^{35}$. Therefore, the patients were divided into four groups: infection one month before chemotherapy, during chemotherapy, six months after chemotherapy, and no infection. A total of 122 had infections within one month before chemotherapy, 158 during induction chemotherapy, 187 within six months after induction chemotherapy, and three had no infection (of 301 patients). The increased platelet count after chemotherapy was associated with complete remission of AML patients. However, there was no correlation between infection and prognosis. Therefore, the infection does not promote platelet production in and it is not a risk factor for AML prognosis.

$\mathrm{CR}$ is characterized by bone marrow blast cell count $\leq 5 \%$, peripheral blood tri-lineage cell recovery, no white blood cells in the classification, no symptoms and signs caused by leukemia infiltration. In this study, 166 cases achieved CR, 39 cases achieved partial remission, 76 cases had no remission, and 20 cases died after one chemotherapy course (301 patients). Roland et al. showed ${ }^{12}$ that some patients had bone marrow blast counts $\leqq 5 \%$, but their platelet counts did not increase after a chemotherapy course in AML patients. Besides, they had a significantly poor prognosis than the CR patients and a relatively better prognosis than the drug-resistant patients. This study showed that patients with elevated platelet counts had a better prognosis than those without platelet counts after the chemotherapy course. The remission rate increased with platelet increase. A chemotherapy course increases platelet count, indicating the response of AML patients to chemotherapy and the recovery of bone marrow hematopoietic function, improving the survival of patients. However, its specific mechanism needs further study.

Therefore, platelet count increase in AML patients after a chemotherapy course is an independent risk factor affecting relief and can be used to evaluate the sensitivity of patients to chemotherapy.

MYH10 deficiency has been reported in many diseases ${ }^{36-39}$. MYH10 is the major transcription factor that causes platelet abnormalities ${ }^{40}$. This is the first study to discuss MYH10 expression in AML, its influence on platelet count, and its prognostic value. This study could contribute to existing knowledge, improve treatment design and improve the prognostic accuracy of AML patients.

The inadequate RUNX1 haploid function, increased bleeding score, abnormal platelet aggregation, and mutations and increased MYH10 in platelets, delayed wound healing, and inherited platelet dysfunction significantly increases the risk of bleeding in FPD/AML ${ }^{41}$.

Recently, several studies have shown that defects in transcription factors that regulate megakaryocyte and platelet production cause hereditary platelet dysfunction. Germline gene mutations encoding the 
hematopoietic transcription factors, RUNX1, GATA-1, FLI1, GFI1b, and ETV6, are associated with quantitative and qualitative platelet abnormalities and variable bleeding symptoms. Some transcription factor defects are also associated with susceptibility to hematological malignancies (RUNX1, ETV6), abnormal erythropoiesis (GATA-1, GFI1b, ETV6), and abnormal immune function (FLI1). The increased MYH10 expression in platelets is a marker for FLI1 and RUNX1 deficiency. The characterization of transcription factor defects causing abnormal platelet function and the gene characteristics leading to differential regulation improves understanding of the role of these genes in platelet formation and function 24,40 .

Mechanical reactivity is a hallmark of pancreatic cancer cells. The mechanical response proteins, MYH9 and MYH14, a-actin 4, and fibrin B, are highly expressed in pancreatic cancer. MYH14 promotes metastatic behavior, 4-hydroxyacetophenone (4-HAP) increases MYH14 assembly, hardening the cells, and 4-HAP minimizes tumor spread. The mechanoresponsive protein is up-regulated with pancreatic cancer progression, indicating that it can improve the survival rate of pancreatic cancer patients by targeting the cytoskeleton. Besides, this protein system can pharmacologically target to inhibit pancreatic cancer and the metastatic potential of cells ${ }^{39}$.

Further research is needed to determine whether $\mathrm{MYH10}$, a mechanical response protein, also has the same effect in AML.

\section{Conclusion}

Increased platelet count in AML patients after chemotherapy was an independent risk factor affecting complete remission. The platelet count also had some guiding significance for evaluating the sensitivity of patients to chemotherapy. MYH10 causes thrombocytopenia in acute myeloid leukemia via RUNX1 gene alteration and influence of prognostic factors. MYH10 variant detection improves the identification of AML molecular characteristics and its prognostic impact on $A M L$, helping in the response analysis to chemotherapeutic agents and further treatment decisions.

\section{Methods}

1. Cases and methods

\subsection{Clinical data}

A total of 301 newly treated AML patients (except M3) admitted to the Fujian Medical University Union Hospital between 2011 to 2017 were used. All patients were diagnosed using bone marrow morphology, immunology, cytogenetics, and molecular biology (MICM) following the 1999 WHO diagnostic criteria 42. Patients who survived at least 30 days after chemotherapy were included in the analysis.

\subsection{Observation indicators}


Patient characteristics, including age, gender, risk category based on cytogenetics/mutation analysis following the European Leukemia Network ${ }^{43}$, culture-proven infection $(\leq 1$ month before induction therapy, during chemotherapy, and $\leq 6$ after chemotherapy course), whether there is platelet transfusion during chemotherapy, bleeding, remission status after treatment course (percentage of bone marrow cells $\leq 5 \%$ ), platelet count on the first day after chemotherapy course, and the highest platelet count after chemotherapy course were observed. The outcome after one chemotherapy course was divided into four groups: remission, partial remission, no remission, and death.

\subsection{Statistical processing}

SPSS software was used for univariate analysis, logistic multiple regression analysis, and cox regression analysis. Univariate and multivariate analyses were performed on age, gender, culture-proven infection, platelet count, and chemotherapy regimen. In univariate analysis, the outcome after chemotherapy was divided into remission (complete remission) and unremission (partial remission, unremission, and death).

\section{Data and methods}

\subsection{Chip data}

GSE12662 was downloaded from the GEO database (https://www.ncbi.nlm.nih.gov/geo/). GSE12662 was based on the GPL570 platform ([HG-U133_Plus_2] Affymetrix Human Genome U133 Plus 2.0 Array). Five FLT3-ITD positive, normal karyotype AML samples (GSM262047, GSM262120, GSM262062, GSM262063, GSM262064) and 22 FLT3-ITD negative, normal karyotype AML samples (GSM262030, GSM262035, GSM262037, GSM262040, GSM262043, GSM262045, GSM262050, GSM262054, GSM262056, GSM262058, GSM262059, GSM262070, GSM262072, GSM262078, GSM262079, GSM262091, GSM262093, GSM262098, GSM262123, GSM262217, GSM262218, GSM262221) and five normal samples (GSM317736, GSM317739, GSM317794, GSM317933, GSM317934) from GSE12662 were compared. The R software was then used to evaluate the data quality from the two data sets.

\subsection{Identification of differentially expressed genes}

GEO2R (http://www.ncbi.nlm.nih.gov/geo/geo2r) was used to screen abb. GEO2R is an intelligent network device used to compare at least two data sets in the GEO series to distinguish differentially expressed genes. The adjusted P-value (adj.P) and the false discovery rate of Benamini and Hochberg were used to achieve a reconciliation between the measurable and great quality of disclosure and the barriers to false positives. The probe sets without quality images or with quality not more than one were emptied or averaged. Herein, the critical point of adj.P $<0.01$ and $|\log F C|>2$ were used to screen and select differentially expressed genes. R ggplot2 was used to draw the volcano and heat maps to show the differentially expressed genes.

2.3 WebGestalt gene set enrichment analysis 
WebGestalt (Web-based Gene SeT AnaLysis Toolkit, http://www.webgestalt.org) is commonly used for functional enrichment analysis ${ }^{44}$. The webgestalt was used to analyze gene ontology (GO) of differentially expressed genes and Kyoto Encyclopedia of Genes and Genomes (KEGG). R ggplot2 package was used to graphically illustrate the GO and KEGG. P $<0.01$ was considered statistically significant.

\subsection{GSEA analysis}

The gene set enrichment analysis (GSEA) method was used to reconfirm the enrichment of the KEGG pathway by analyzing the differentially expressed data in the GSEA software version 4.0 with molecular signature database (MSigDB v7.1) Of predefined gene sets ${ }^{45}$. The related KEGG pathway results (cancer pathway) were then used for further analysis.

\subsection{PPI network construction}

The online gene retrieval tool (STRING; http://STRING-db.org) (version 11.0) ${ }^{46}$ was used to construct a protein-protein interaction (PPI) network of 62 common differentially expressed genes to visualize potential gene interaction networks. Various online protein tools have been used to reveal related genes involved in cancer pathways. Furthermore, the tools can identify the common genes involved in cancer pathways and transcriptional disorders in cancer and their potential interactions in the two animal models.

\subsection{GEPIA database}

Gene Expression Profiling Interactive Analysis (GEPIA) database (http://gepia.cancer-pku.cn/) is a newly developed interactive Web server ${ }^{47}$. GEPIA uses standard processing pipelines to analyze cancer genomes, RNA sequencing expression data of 9,736 tumors, and 8,587 normal samples of the Atlas and Genotype Tissue Expression (GTEx) project. GEPIA provides customizable functions, including tumor/normal differential expression analysis, cancer type or pathological stage analysis, patient survival analysis, similar gene detection, correlation, and dimensionality reduction analyses.

\section{List Of Dreviations}




\begin{tabular}{|ll|}
\hline Acute myeloid leukemia & AML \\
\hline Complete remission & CR \\
\hline Morphological leukemia-free state & MLFS \\
\hline Absolute neutrophil count & ANC \\
\hline Platelets & PLT \\
\hline Principal component analysis & PCA \\
\hline Biological processes & BP \\
\hline Molecular functions & MF \\
\hline Cellular components & CC \\
\hline Megakaryocytes & MKs \\
\hline Myosin heavy chain IIA & MYH9 \\
\hline Myosin heavy chain IIB & MYH10 \\
\hline Leukemic bone marrow & LAML \\
\hline Chronic myeloid leukemia & CML \\
\hline Acute lymphocytic leukemia & ALL \\
\hline Gene ontology & GO \\
\hline Kyoto Encyclopedia of Genes and Genomes & KEGG \\
\hline Gene set enrichment analysis & GSEA \\
\hline Protein-protein interaction & PPI \\
\hline Genotype Tissue Expression & GTEx \\
\hline Gene Expression Profiling Interactive Analysis & GEPIA \\
\hline
\end{tabular}

Supplementary Information:

Additional Fig. 1.

\section{Declarations}

Ethics approval and consent to participate:

Not applicable.

Consent for publication: 
Not applicable.

Availability of data and materials:

All data generated or analyzed during this study are included in this published article, and its supplementary information files, or upon a reasonable request. The public data set was used in this paper and can be found at:

GEO database: https://www.ncbi.nlm.nih.gov/geo/

GEO2R: http://www.ncbi.nlm.nih.gov/geo/geo2r

WebGestalt: http://www.webgestalt.org

STRING: http://STRING-db.org

GEPIA database: http://gepia.cancer-pku.cn/

Competing interests:

Not applicable.

\section{Funding:}

This study is supported by National Natural Science Foundation of China (81800136), Joint Funds for the innovation of science and Technology, Fujian province (2017Y9097), Fujian Natural Science Joint Program (2018J01181), Program for New Century Excellent Talents in University (2018B048), The government-funded project of the construction of high-level laboratory (Min2017 No.4 File), Construction project of Fujian medical center of hematology (Min201704) and National and Fujian Provincial Key Clinical Specialty Discipline Construction Program, P. R.C.

\section{Authors' contributions:}

Qirong Xiao, Bicun Lin, Kaiting Lin and Xiaobin Lin collected the data, performed the analysis, and wrote the paper. Ping Chen conceived and designed the analysis, contributed analysis tools, supervised, acquired funding, reviewed, and edited the paper. All authors read and approved the final manuscript.

\section{Acknowledgements:}

Not applicable.

\section{References}

1. Khwaja, A. et al. Acute myeloid leukaemia. Nature reviews. Disease primers 2, 16010, doi:10.1038/nrdp.2016.10 (2016). 
2. Anderlini, P. et al. Factors predicting complete remission and subsequent disease-free survival after a second course of induction therapy in patients with acute myelogenous leukemia resistant to the first. Leukemia 10, 964-969 (1996).

3. Wheatley, K. et al. A simple, robust, validated and highly predictive index for the determination of riskdirected therapy in acute myeloid leukaemia derived from the MRC AML 10 trial. United Kingdom Medical Research Council's Adult and Childhood Leukaemia Working Parties. British journal of haematology 107, 69-79, doi:10.1046/j.1365-2141.1999.01684.x (1999).

4. Rowe, J. M. et al. Adult patients with acute myeloid leukemia who achieve complete remission after 1 or 2 cycles of induction have a similar prognosis: a report on 1980 patients registered to 6 studies conducted by the Eastern Cooperative Oncology Group. Cancer 116, 5012-5021, doi:10.1002/cncr.25263 (2010).
5. et al.
$\% \mathrm{~J}$
36, 89-94 (2015).

6. Mauritzson, N. et al. A single-center population-based consecutive series of 1500 cytogenetically investigated adult hematological malignancies: karyotypic features in relation to morphology, age and gender. European journal of haematology 62, 95-102, doi:10.1111/j.1600-0609.1999.tb01728.x (1999).

7. Schoch, C. et al. The influence of age on prognosis of de novo acute myeloid leukemia differs according to cytogenetic subgroups. Haematologica 89, 1082-1090 (2004).

8. Bacher, U. et al. Population-based age-specific incidences of cytogenetic subgroups of acute myeloid leukemia. Haematologica 90, 1502-1510 (2005).

9. Creutzig, U. et al. Changes in cytogenetics and molecular genetics in acute myeloid leukemia from childhood to adult age groups. Cancer 122, 3821-3830, doi:10.1002/cncr.30220 (2016).

10. Sievers, E. L. et al. Efficacy and safety of gemtuzumab ozogamicin in patients with CD33-positive acute myeloid leukemia in first relapse. Journal of clinical oncology : official journal of the American Society of Clinical Oncology 19, 3244-3254, doi:10.1200/jco.2001.19.13.3244 (2001).

11. Cheson, B. D. et al. Revised recommendations of the International Working Group for Diagnosis, Standardization of Response Criteria, Treatment Outcomes, and Reporting Standards for Therapeutic Trials in Acute Myeloid Leukemia. Journal of clinical oncology : official journal of the American Society of Clinical Oncology 21, 4642-4649, doi:10.1200/jco.2003.04.036 (2003).

12. Walter, R. B. et al. Effect of complete remission and responses less than complete remission on survival in acute myeloid leukemia: a combined Eastern Cooperative Oncology Group, Southwest Oncology Group, and M. D. Anderson Cancer Center Study. Journal of clinical oncology : official journal of the American Society of Clinical Oncology 28, 1766-1771, doi:10.1200/jco.2009.25.1066 (2010).

13. Döhner, H. et al. Diagnosis and management of acute myeloid leukemia in adults: recommendations from an international expert panel, on behalf of the European LeukemiaNet. Blood 115, 453-474, doi:10.1182/blood-2009-07-235358 (2010). 
14. Chen, X. et al. Relation of clinical response and minimal residual disease and their prognostic impact on outcome in acute myeloid leukemia. Journal of clinical oncology : official journal of the American Society of Clinical Oncology 33, 1258-1264, doi:10.1200/jco.2014.58.3518 (2015).

15. Bluteau, D. et al. Regulation of megakaryocyte maturation and platelet formation. Journal of thrombosis and haemostasis : JTH7 Suppl 1, 227-234, doi:10.1111/j.1538-7836.2009.03398.x (2009).

16. Ravid, K., Lu, J., Zimmet, J. M. \& Jones, M. R. Roads to polyploidy: the megakaryocyte example. Journal of cellular physiology 190, 7-20, doi:10.1002/jcp.10035 (2002).

17. Zimmet, J. \& Ravid, K. Polyploidy: occurrence in nature, mechanisms, and significance for the megakaryocyte-platelet system. Experimental hematology 28, 3-16, doi:10.1016/s0301472x(99)00124-1 (2000).

18. Roy, A. et al. Activity of nonmuscle myosin II isoforms determines localization at the cleavage furrow of megakaryocytes. Blood 128, 3137-3145, doi:10.1182/blood-2016-04-711630 (2016).

19. Lordier, L. et al. RUNX1-induced silencing of non-muscle myosin heavy chain IIB contributes to megakaryocyte polyploidization. Nature communications 3, 717, doi:10.1038/ncomms1704 (2012).

20. Tijssen, M. R. \& Ghevaert, C. Transcription factors in late megakaryopoiesis and related platelet disorders. Journal of thrombosis and haemostasis : JTH 11, 593-604, doi:10.1111/jth.12131 (2013).

21. Connelly, J. P. et al. Targeted correction of RUNX1 mutation in FPD patient-specific induced pluripotent stem cells rescues megakaryopoietic defects. Blood 124, 1926-1930, doi:10.1182/blood2014-01-550525 (2014).

22. Kuo, M. C. et al. RUNX1 mutations are frequent in chronic myelomonocytic leukemia and mutations at the C-terminal region might predict acute myeloid leukemia transformation. Leukemia 23, 14261431, doi:10.1038/leu.2009.48 (2009).

23. Bluteau, D. et al. Dysmegakaryopoiesis of FPD/AML pedigrees with constitutional RUNX1 mutations is linked to myosin II deregulated expression. Blood 120, 2708-2718, doi:10.1182/blood-2012-04422337 (2012).

24. Antony-Debré, I. et al. MYH10 protein expression in platelets as a biomarker of RUNX1 and FLI1 alterations. Blood 120, 2719-2722, doi:10.1182/blood-2012-04-422352 (2012).

25. Zhang, Q. et al. Pretreatment platelet count predicts survival outcome of patients with de novo nonM3 acute myeloid leukemia. PeerJ 5, e4139, doi:10.7717/peerj.4139 (2017).

26. Bănescu, C. et al. Cytokine rs361525, rs1800750, rs1800629, rs1800896, rs1800872, rs1800795, rs1800470, and rs2430561 SNPs in relation with prognostic factors in acute myeloid leukemia. Cancer medicine 8, 5492-5506, doi:10.1002/cam4.2424 (2019).

27. Ornstein, M. C. et al. Predictive factors for latency period and a prognostic model for survival in patients with therapy-related acute myeloid leukemia. American journal of hematology 89, 168-173, doi:10.1002/ajh.23605 (2014). 
28. Zhang, Y. et al. Low Platelet Counts at Diagnosis Predict Better Survival for Patients with Intermediate-Risk Acute Myeloid Leukemia. Acta haematologica 143, 9-18, doi:10.1159/000500230 (2020).

29. Weinberg, O. K. et al. Reproducibility and prognostic significance of morphologic dysplasia in de novo acute myeloid leukemia. Modern pathology : an official journal of the United States and Canadian Academy of Pathology, Inc 28, 965-976, doi:10.1038/modpathol.2015.55 (2015).

30. Gupta, S. et al. Treatment-related mortality in children with acute lymphoblastic leukemia in Central America. Cancer 117, 4788-4795, doi:10.1002/cncr.26107 (2011).

31. Malkan, U. Y. et al. Rebound Thrombocytosis following Induction Chemotherapy is an Independent Predictor of a Good Prognosis in Acute Myeloid Leukemia Patients Attaining First Complete Remission. Acta haematologica 134, 32-37, doi:10.1159/000369917 (2015).

32. Trafalis, D. T. et al. Platelet production and related pathophysiology in acute myelogenous leukemia at first diagnosis: prognostic implications. Oncology reports 19, 1021-1026 (2008).

33. Wang, Y. et al. Clinical value of the quantitation of average daily platelet increase during the recovery period in childhood acute lymphoblastic leukaemia. Platelets 30, 923-926, doi:10.1080/09537104.2018.1548011 (2019).

34. Mangaonkar, A. et al. Prognostic value of complete remission with superior platelet counts in acute myeloid leukemia. The Journal of community and supportive oncology 14, 66-71, doi:10.12788/jcso.0231 (2016).

35. Lu, H. et al. Bone marrow stromal cells transduced with a thrombopoietin, interleukin-6, and interleukin-11 syncretic gene induce cord mononuclear cells to generate platelets in vitro. Transfusion 55, 176-186, doi:10.1111/trf.12800 (2015).

36. Wang, Y. et al. Myosin Heavy Chain 10 (MYH10) Gene Silencing Reduces Cell Migration and Invasion in the Glioma Cell Lines U251, T98G, and SHG44 by Inhibiting the Wnt/ $\beta$-Catenin Pathway. Medical science monitor : international medical journal of experimental and clinical research 24, 9110-9119, doi:10.12659/msm.911523 (2018).

37. Liu, W. et al. MiR-200a Regulates Nasopharyngeal Carcinoma Cell Migration and Invasion by Targeting MYH10. Journal of Cancer 11, 3052-3060, doi:10.7150/jca.40438 (2020).

38. Kim, H. T. et al. Myh10 deficiency leads to defective extracellular matrix remodeling and pulmonary disease. Nature communications 9, 4600, doi:10.1038/s41467-018-06833-7 (2018).

39. Surcel, A. et al. Targeting Mechanoresponsive Proteins in Pancreatic Cancer: 4-Hydroxyacetophenone Blocks Dissemination and Invasion by Activating MYH14. Cancer research 79, 4665-4678, doi:10.1158/0008-5472.Can-18-3131 (2019).

40. Daly, M. E. Transcription factor defects causing platelet disorders. Blood reviews 31, 1-10, doi:10.1016/j.blre.2016.07.002 (2017).

41. Badin, M. S. et al. Molecular phenotype and bleeding risks of an inherited platelet disorder in a family with a RUNX1 frameshift mutation. Haemophilia : the official journal of the World Federation of Hemophilia 23, e204-e213, doi:10.1111/hae.13169 (2017). 
42. Arber, D. A. et al. The 2016 revision to the World Health Organization classification of myeloid neoplasms and acute leukemia. Blood 127, 2391-2405, doi:10.1182/blood-2016-03-643544 (2016).

43. Döhner, H. et al. Diagnosis and management of AML in adults: 2017 ELN recommendations from an international expert panel. Blood 129, 424-447, doi:10.1182/blood-2016-08-733196 (2017).

44. Liao, Y., Wang, J., Jaehnig, E. J., Shi, Z. \& Zhang, B. WebGestalt 2019: gene set analysis toolkit with revamped Uls and APIs. Nucleic acids research 47, W199-w205, doi:10.1093/nar/gkz401 (2019).

45. Subramanian, A. et al. Gene set enrichment analysis: a knowledge-based approach for interpreting genome-wide expression profiles. Proceedings of the National Academy of Sciences of the United States of America 102, 15545-15550, doi:10.1073/pnas.0506580102 (2005).

46. Szklarczyk, D. et al. STRING v11: protein-protein association networks with increased coverage, supporting functional discovery in genome-wide experimental datasets. Nucleic acids research 47, D607-d613, doi:10.1093/nar/gky1131 (2019).

47. Tang, Z. et al. GEPIA: a web server for cancer and normal gene expression profiling and interactive analyses. Nucleic acids research 45, W98-w102, doi:10.1093/nar/gkx247 (2017).

\section{Figures}




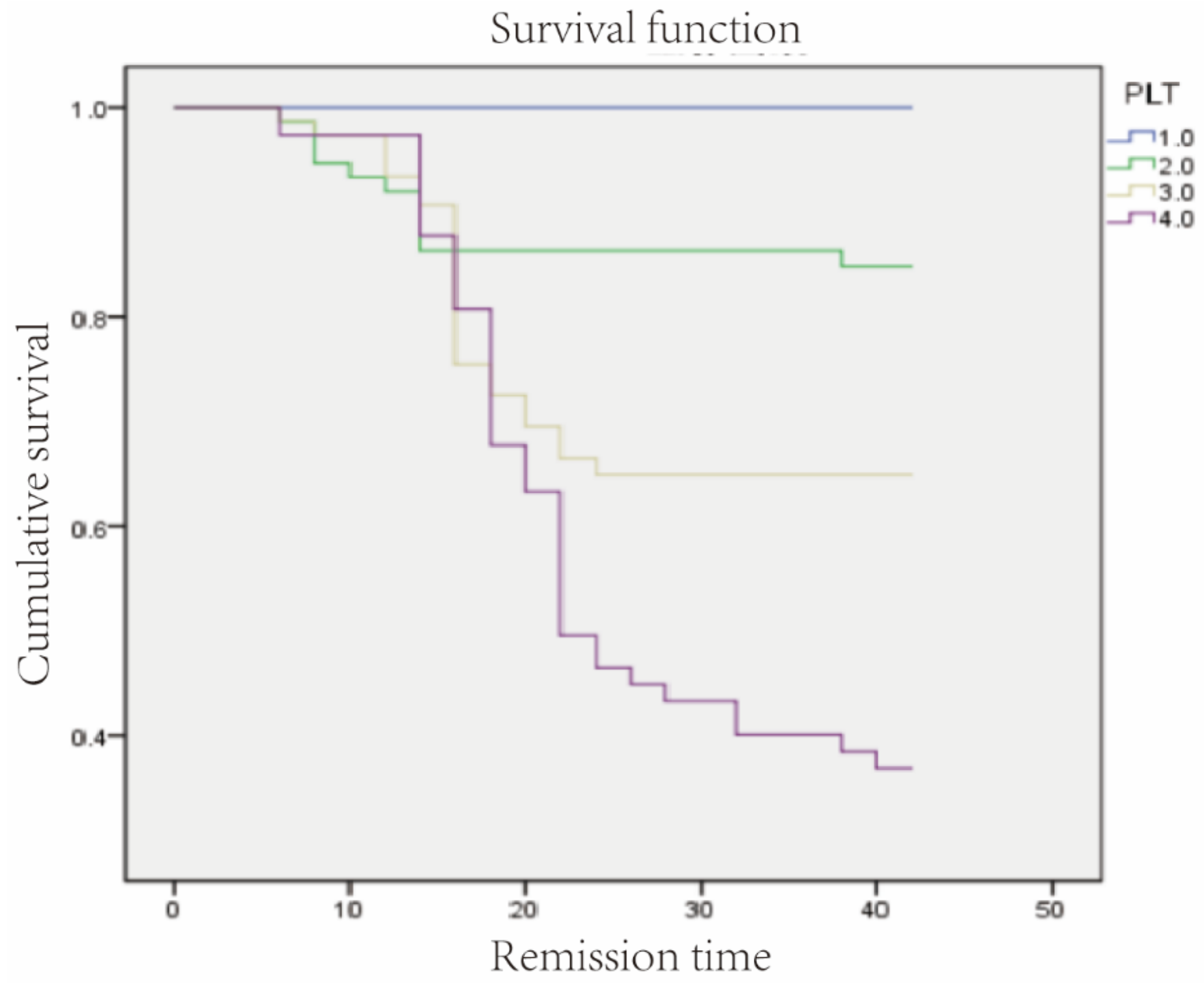

Figure 1

Cumulative remission rate PLT1 represents an increase of platelets $-591-5 \times 109$, PLT2 represents an increase of platelets $6-49 \times 109$, PLT3 represents an increase of platelets $50-116 \times 109$, and PLT4 represents an increase of platelets $117-1184 \times 109$. 
A

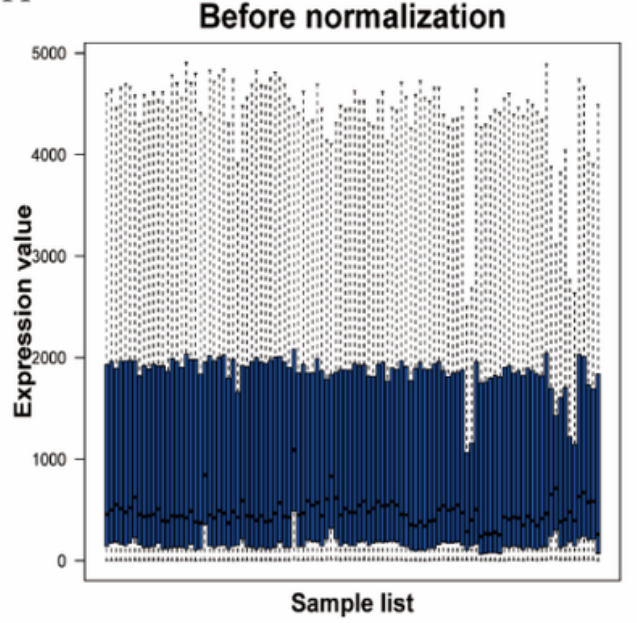

B

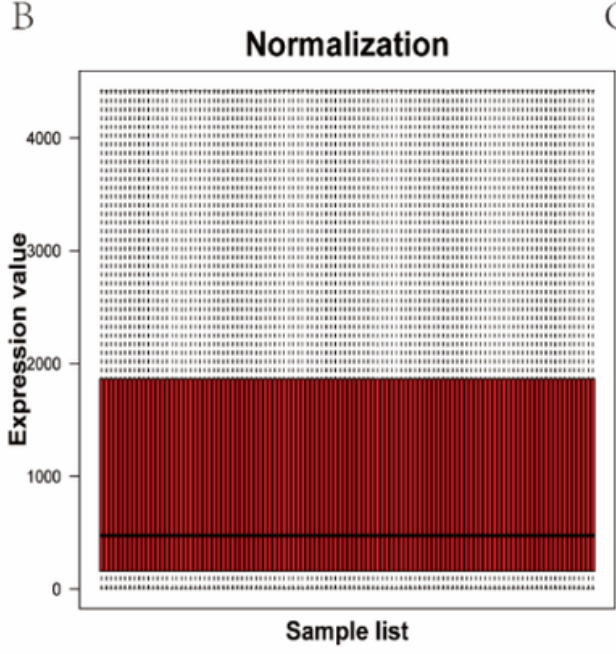

$\mathrm{C}$

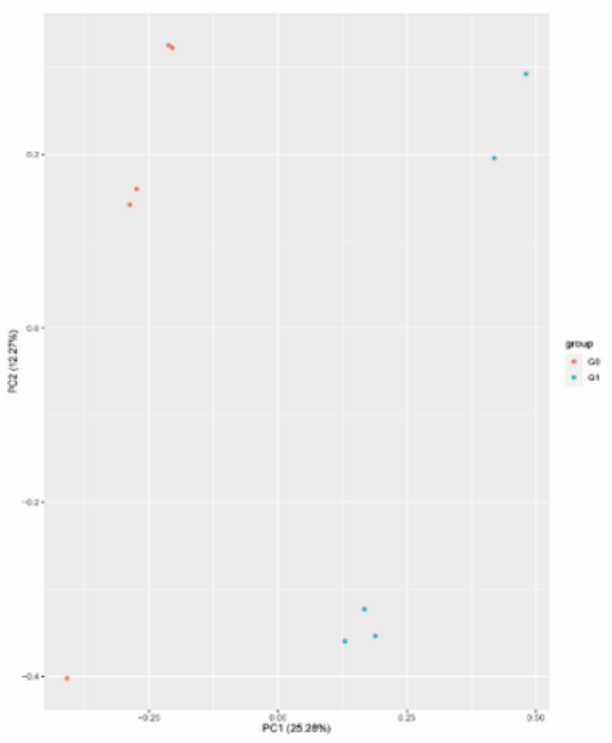

$\mathrm{D}$

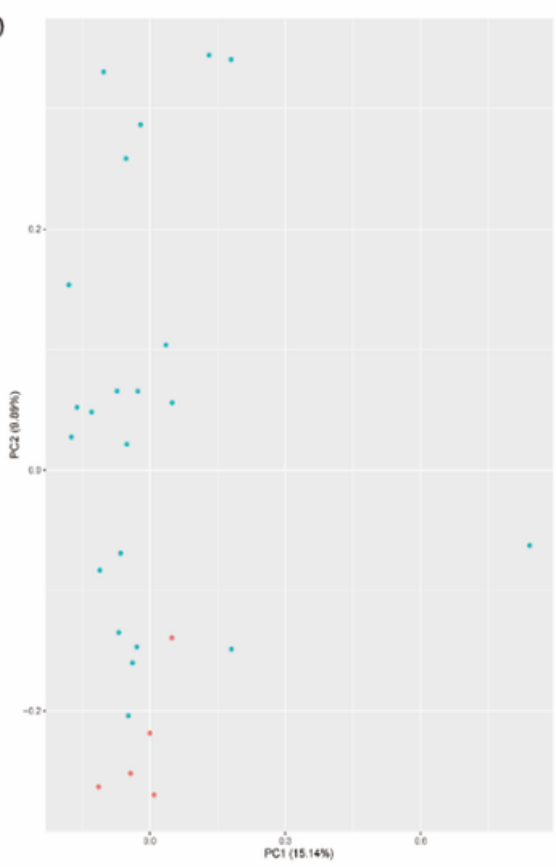

E

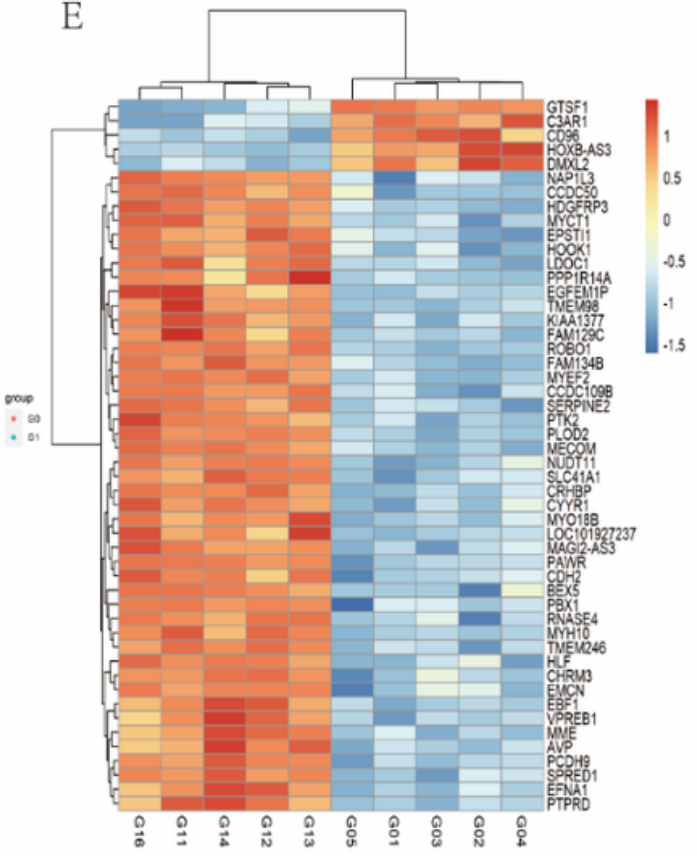

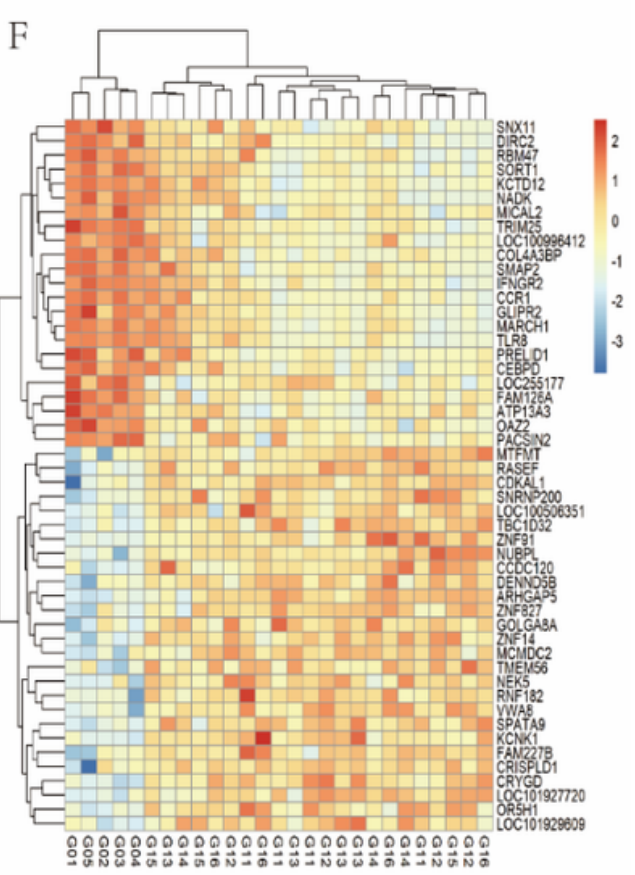

Figure 2

Distribution of gene expression data in each group A and B : Probe of GSE12662 database before and after standardization; $C$ and D Heat map of PCA and TOP50DEGs in FLT3-ITD positive group (G0: the control group, G1: the FLT3-ITD positive group); E and F Heat map of PCA and TOP50DEGs in FLT3-ITD negative group (G0: the control group, G1: the FLT3-ITD negative group). 

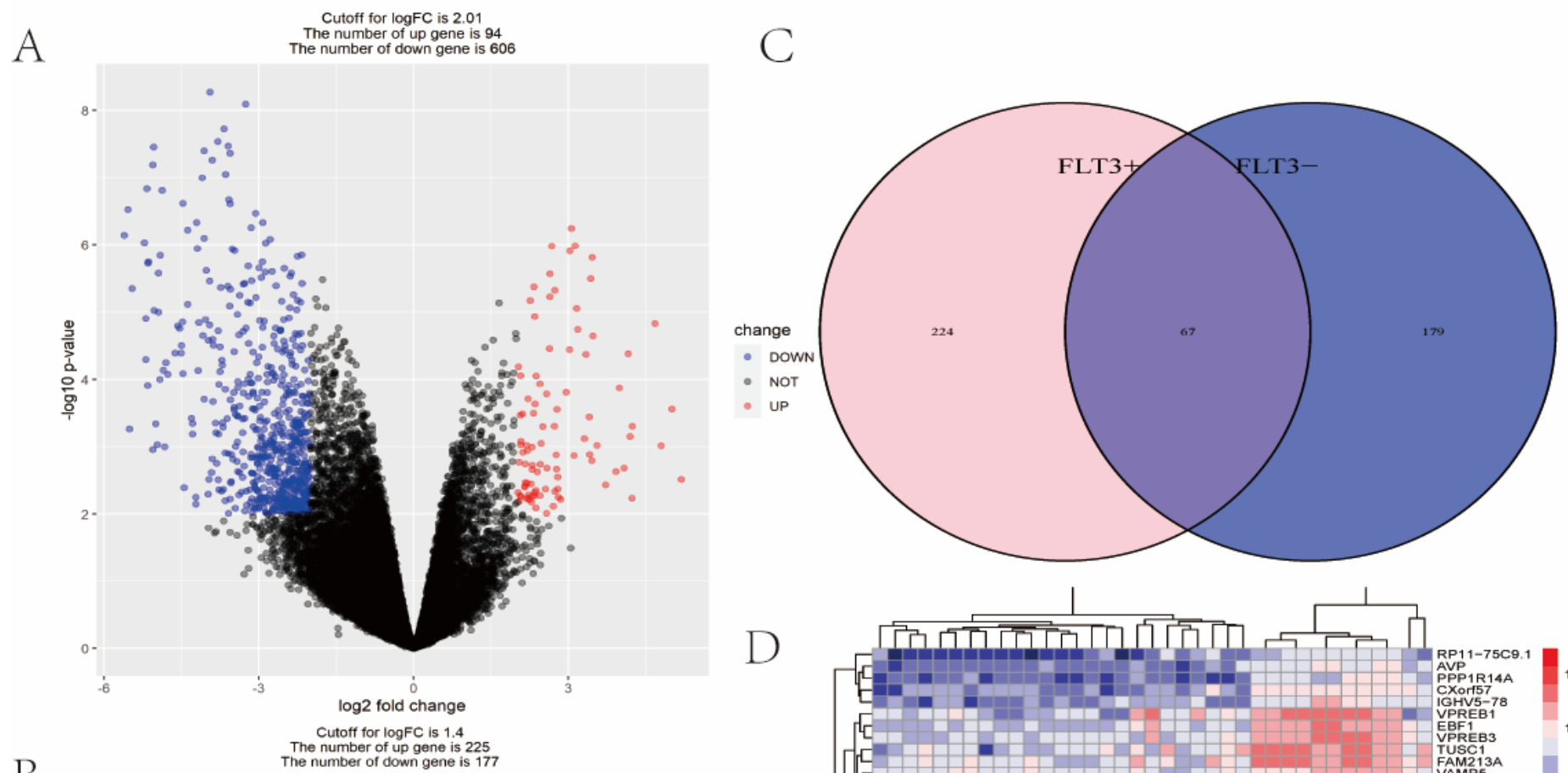

B
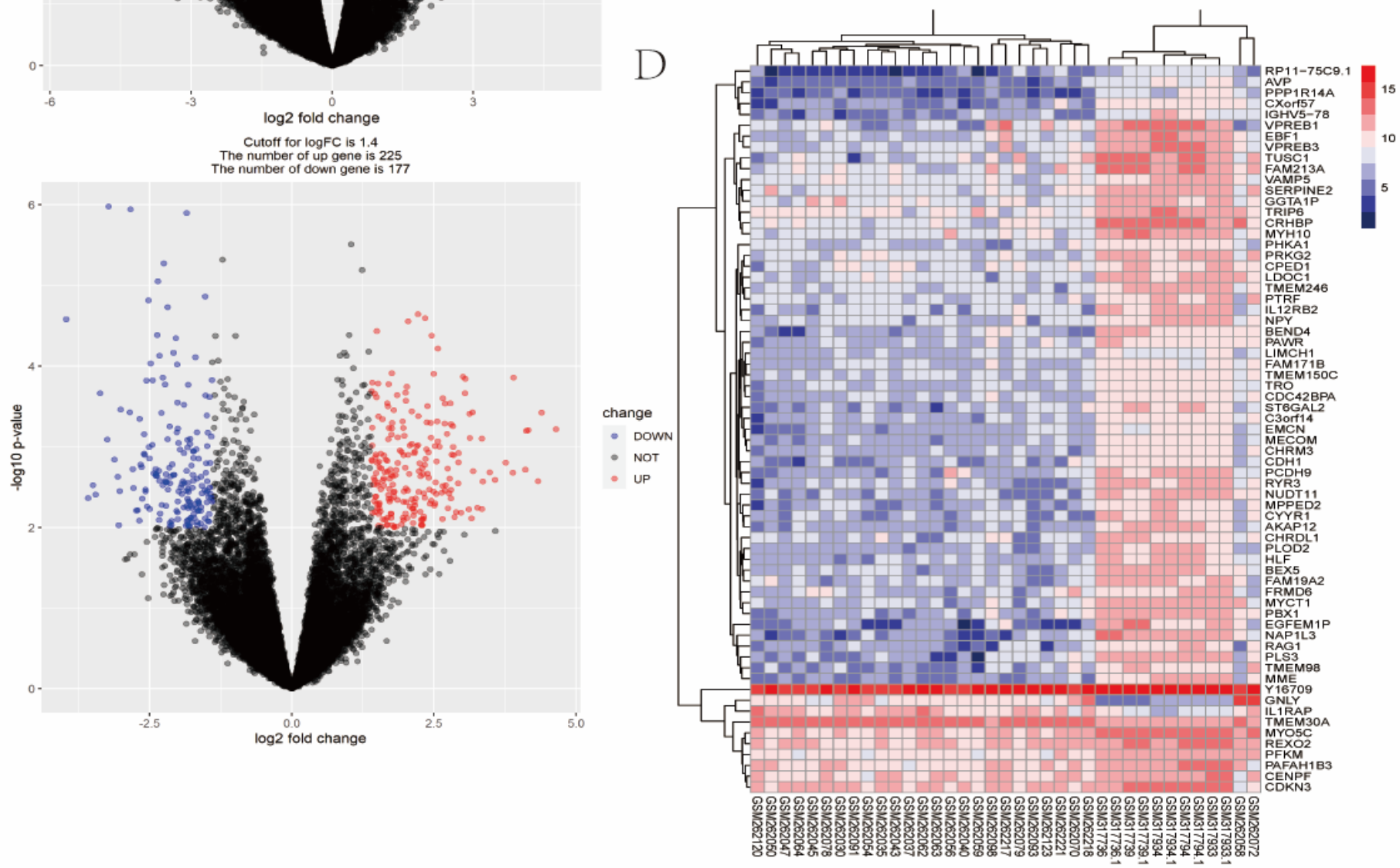

Figure 3

Visualization of differentially expressed genes A: Volcano Plot of DEGs in FLT3-ITD positive group vs control group; B: Volcano Plot of DEGs in FLT3-ITD negative group vs control group; C: Venn diagram of CO-DEGs in FLT3-ITD positive group and FLT3-ITD negative group; D: Heat map of CO-DEGs in FLT3-ITD positive group and FLT3-ITD negative group. 


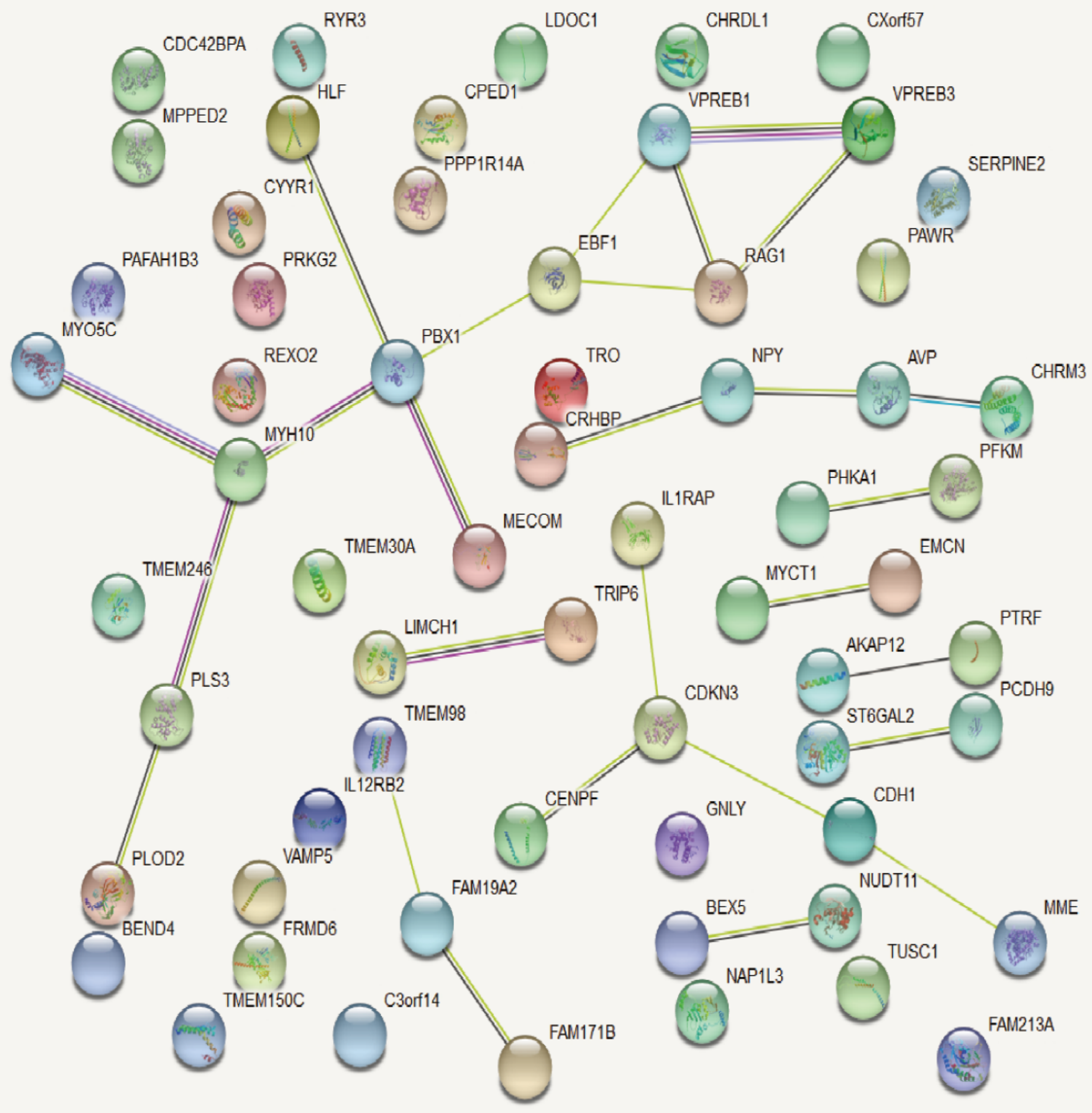

Figure 4

PPI network diagram PPI network of CO-DEGs between FLT3-ITD positive group and FLT3-ITD negative group 
A

Bar chart of Biological Process categories

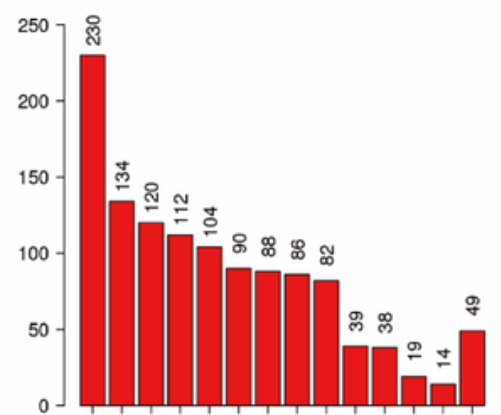

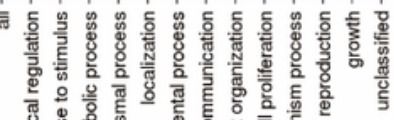

응 윤
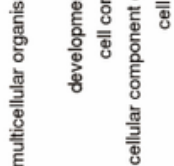

B

Bar chart of Biological Process categories

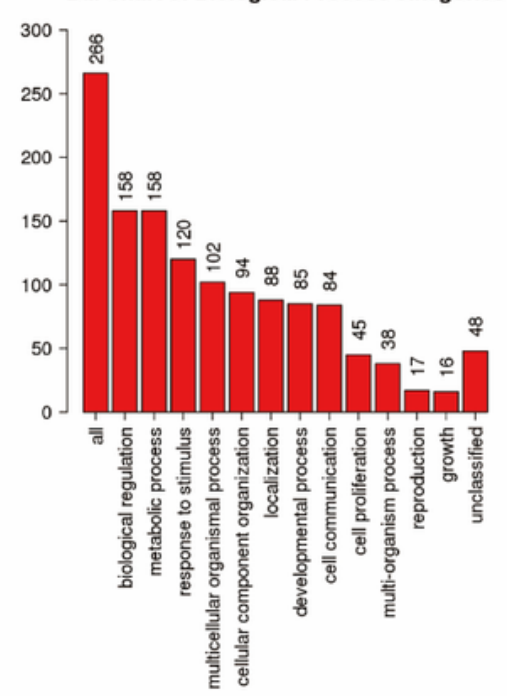

$\mathrm{C}$

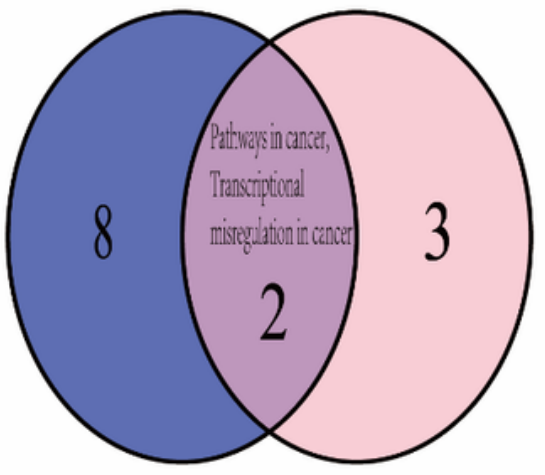

FLT3+
Bar chart of Cellular Component categories

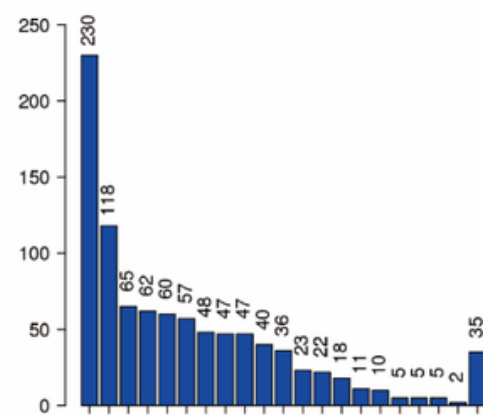

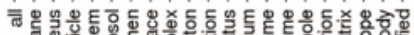
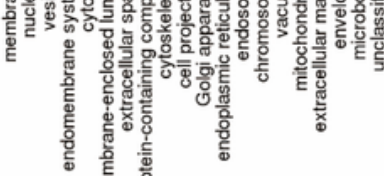

Bar chart of Cellular Component categories

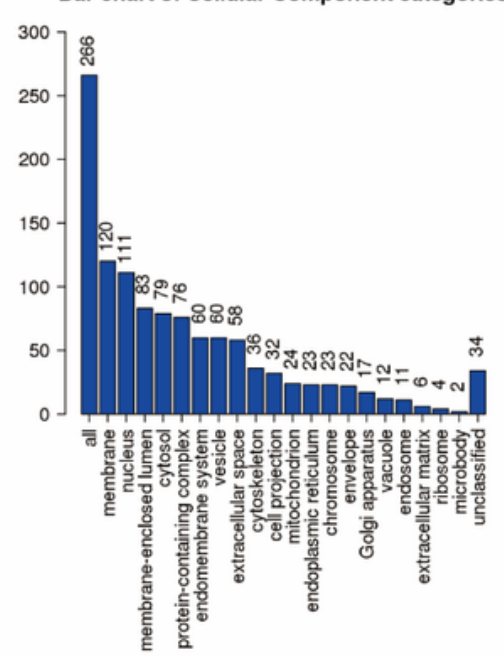

D)

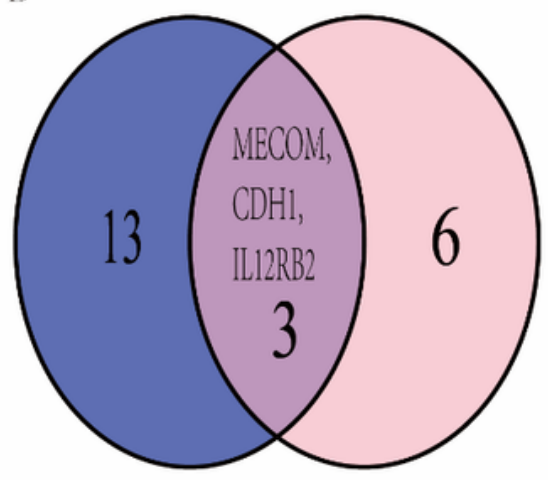

FLT3+
Bar chart of Molecular Function categories

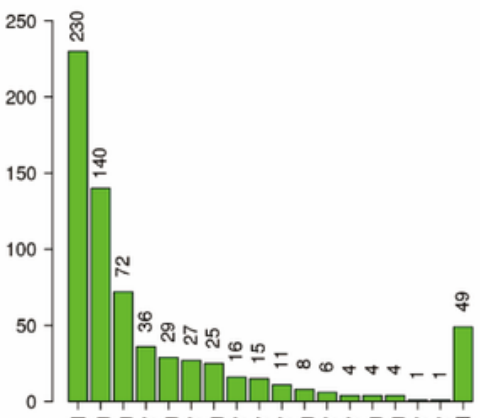

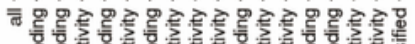

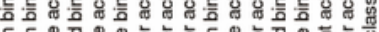

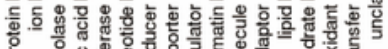

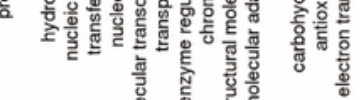

Bar chart of Molecular Function categories

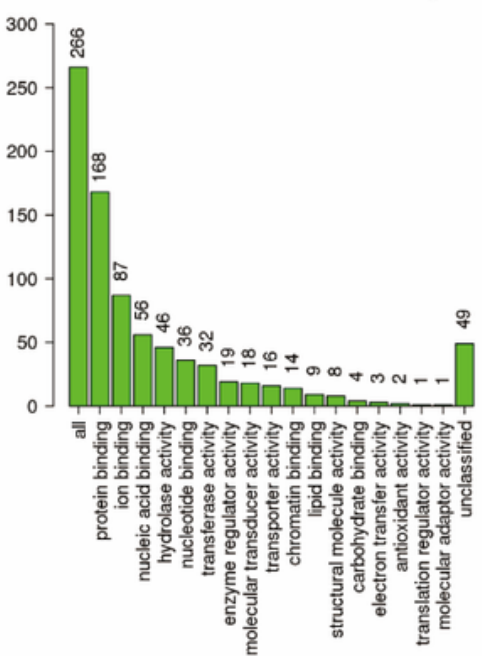

F

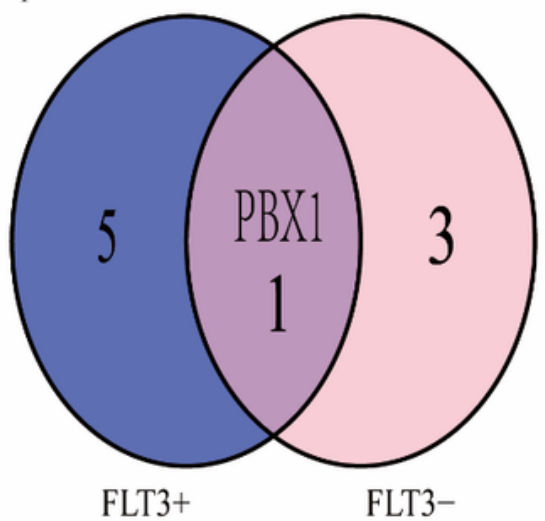

Figure 5

Enrichment analysis of GO function and KEGG pathway A: GO enrichment of DEGs in FLT3-ITD positive group; B: GO enrichment of DEGs in FLT3-ITD negative group; C: Venn diagram of common tumor related KEGG enrichment in FLT3-ITD positive group and FLT3-ITD negative group; D and F: Venn diagram of common CO-DEGs in pathways and transcriptional misregulation in cancer. 


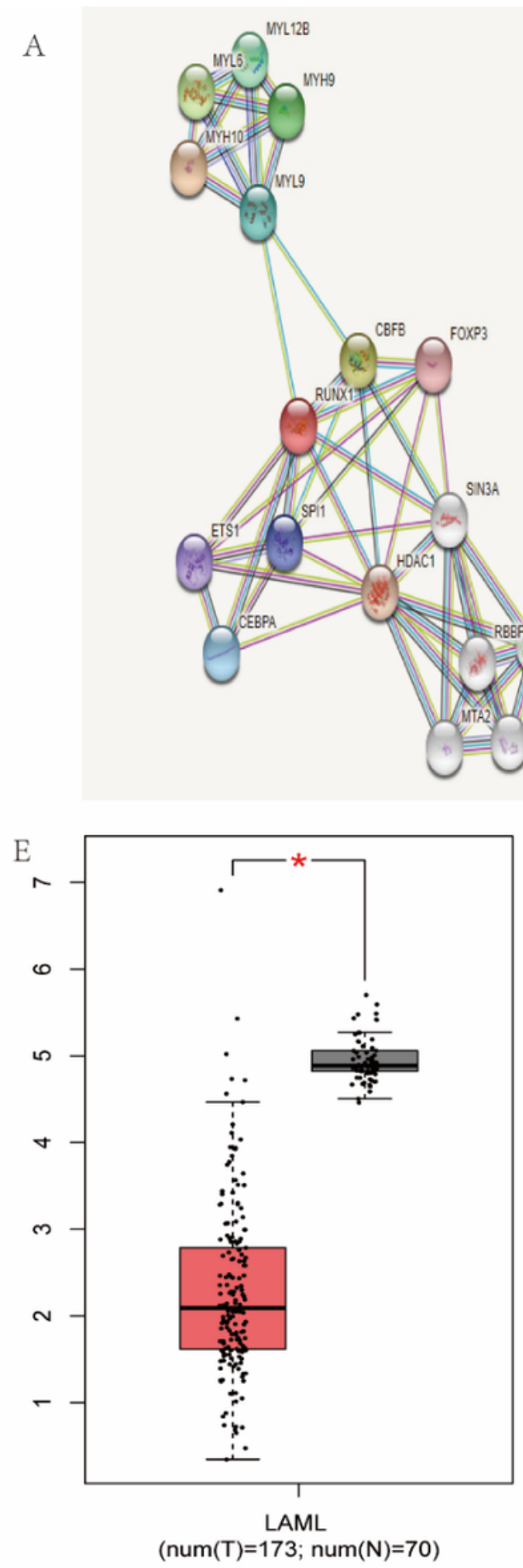

B
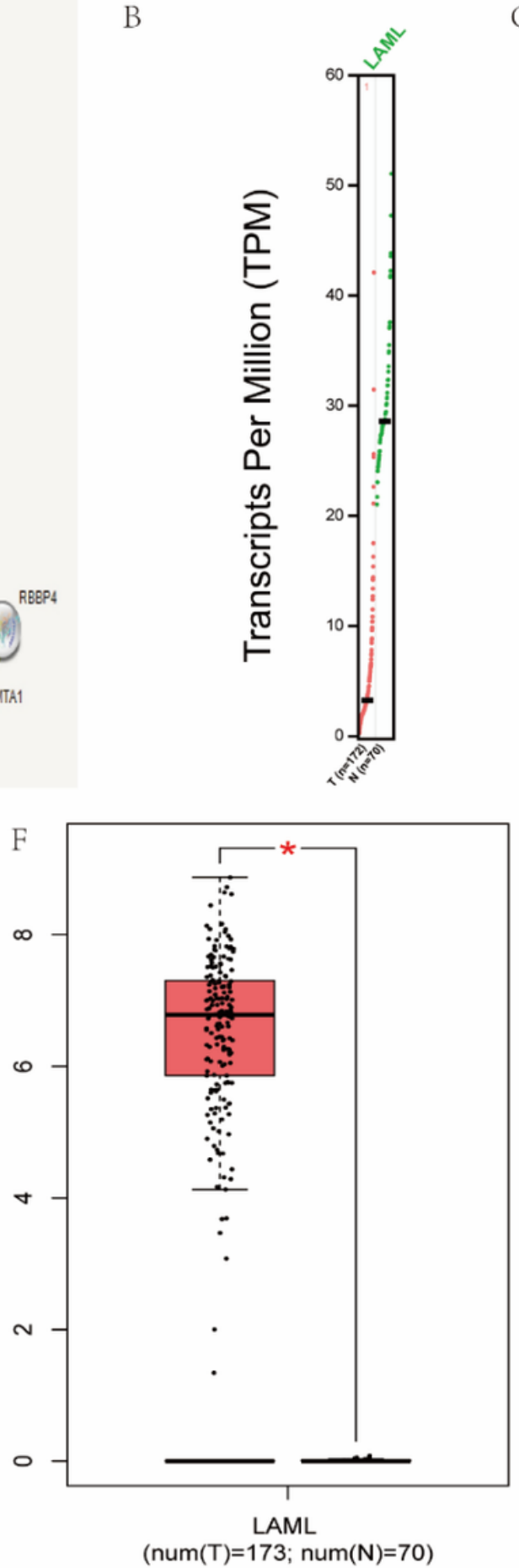

C

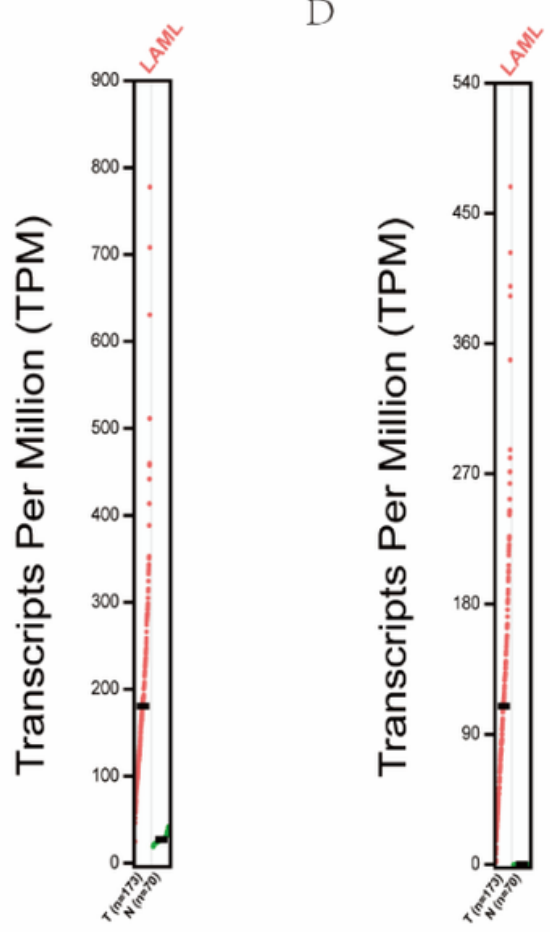

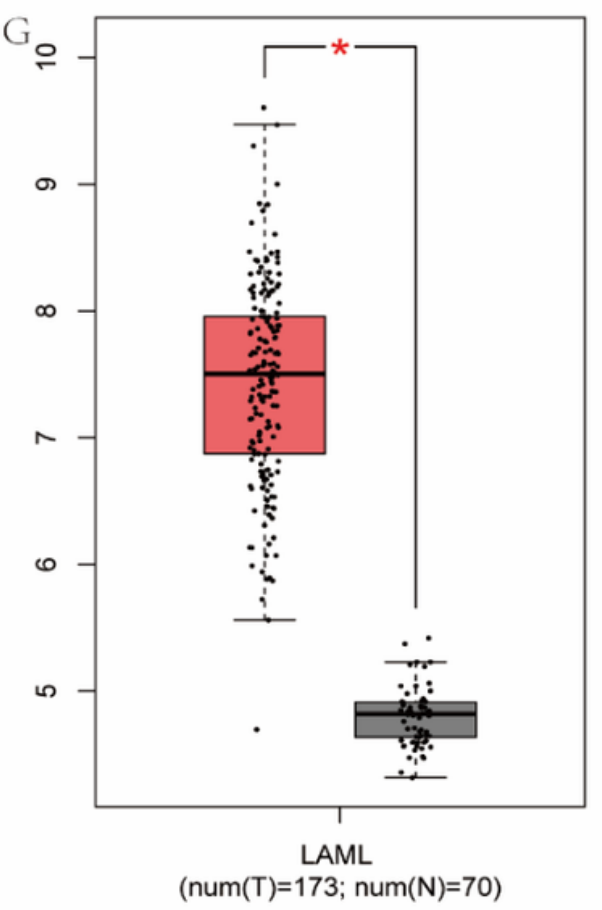

Figure 6

PPI network and mRNA expression map A: PPI network is constructed by MYH10 and RUNX1; B and C and D: Scatter plot of mRNA expression of MYH10 RUNX1 and FLT3 in AML; E and F and G: Box plot of mRNA expression of MYH10 RUNX1 and FLT3 in AML |Log2FC| 1 p-value 0.01 
A

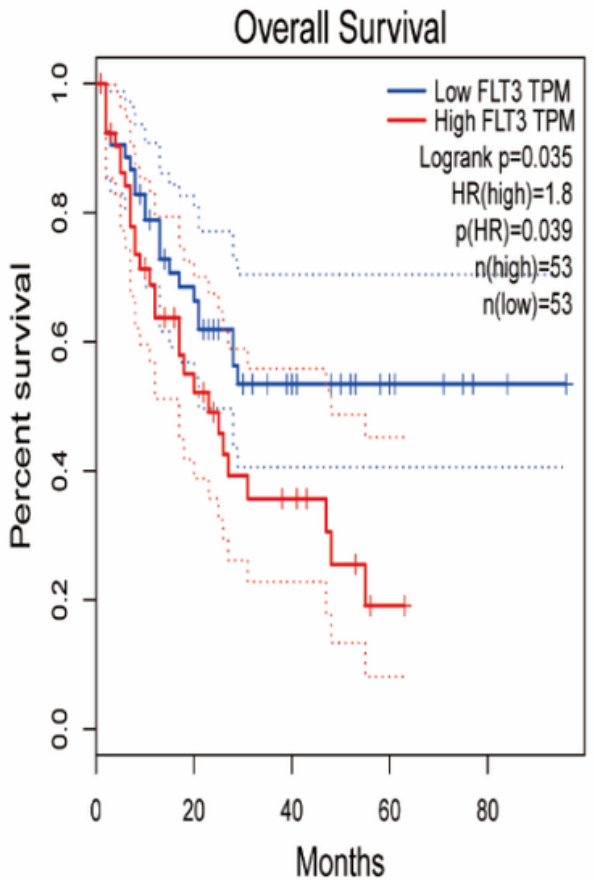

B

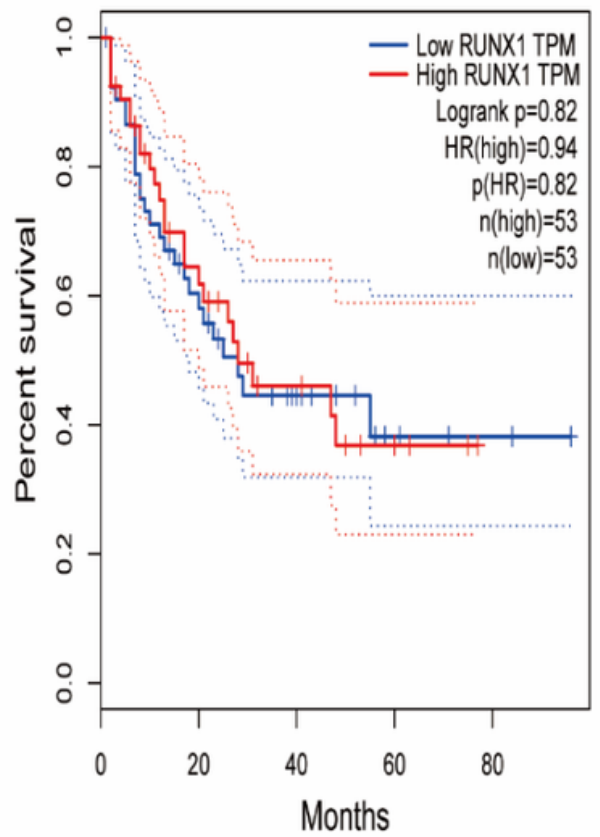

C Overall Survival

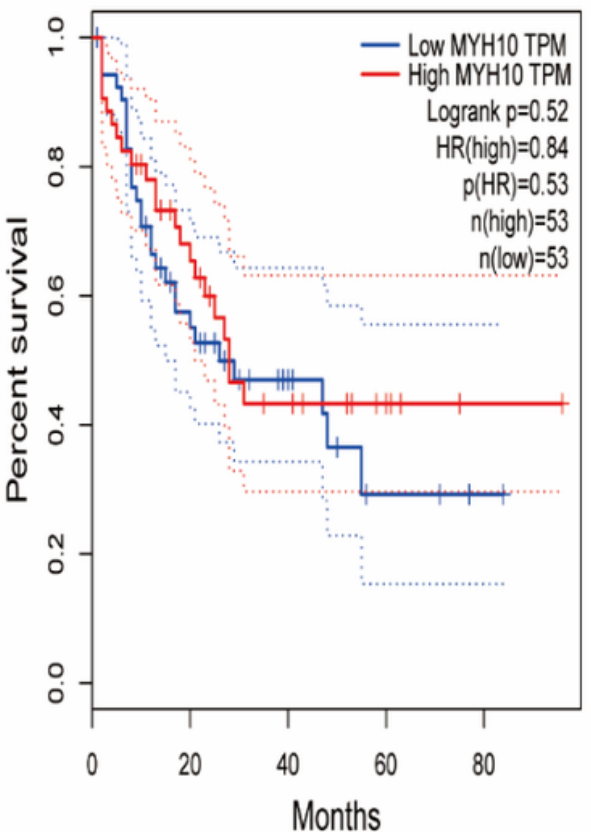

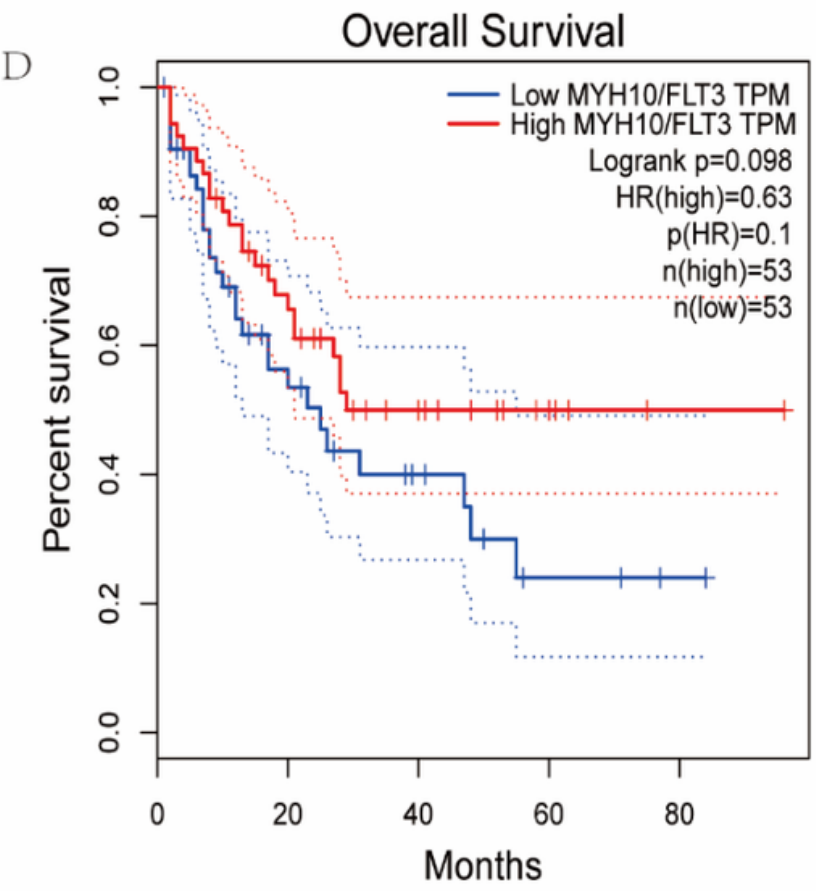

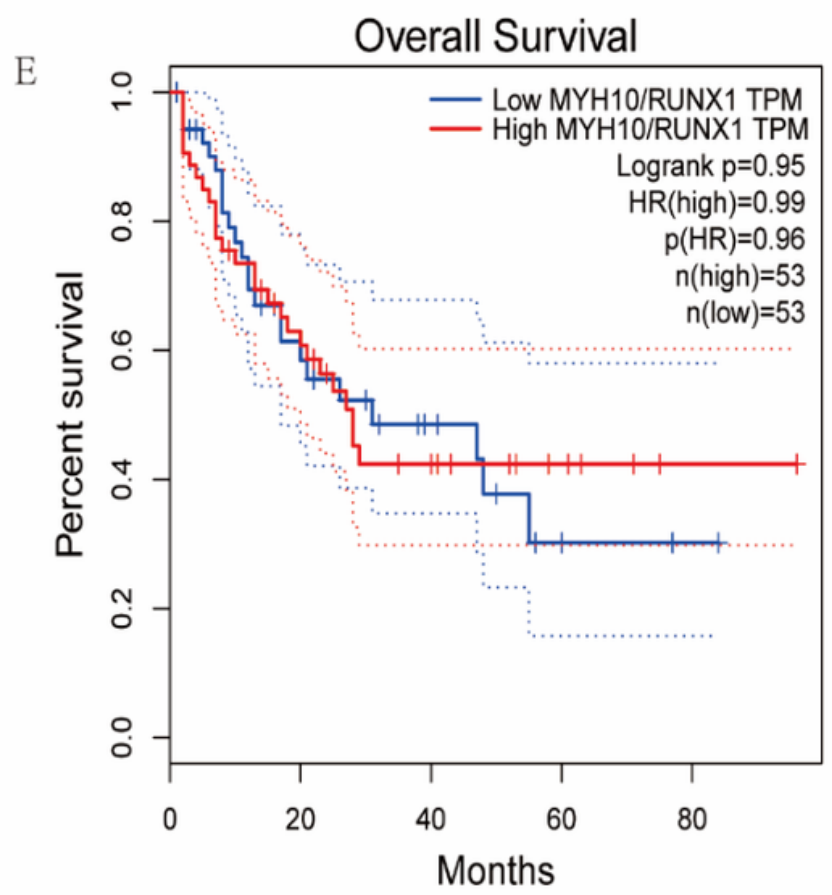

Figure 7

Survival curve A and B and C: Survival curves of MYH10,RUNX1,FLT3 in AML; D and F: Survival curves of MYH10 after RUNX1/FLT3 correction.

\section{Supplementary Files}

This is a list of supplementary files associated with this preprint. Click to download.

- supplementaryfigure.jpg 